Preprint typeset in JHEP style. - PAPER VERSION

ILL-(TH)-00-01

hep-th/0001055

\title{
Discrete Torsion, AdS/CFT and Duality
}

\author{
David Berenstein*and Robert G. Leigh ${ }^{\dagger}$ \\ Department of Physics \\ University of Illinois at Urbana-Champaign \\ Urbana, IL 61801
}

\begin{abstract}
We analyse D-branes on orbifolds with discrete torsion, extending earlier results. We analyze certain Abelian orbifolds of the type $\mathbb{C}^{3} / \Gamma$, where $\Gamma$ is given by $\mathbb{Z}_{m} \times \mathbb{Z}_{n}$, for the most general choice of discrete torsion parameter. By comparing with the AdS/CFT correspondence, we can consider different geometries which give rise to the same physics. This identifies new mirror pairs and suggests new dualities at large $N$. As a by-product we also get a more geometric picture of discrete torsion.
\end{abstract}

KEywords: Discrete Torsion,Duality in Gauge Field Theories,D-branes,Brane Dynamics in Gauge Theories.

* berenste@pobox.hep.uiuc .edu

$\dagger \sim$ rgleigh@uiuc .edu 


\section{Contents}

1. Introduction 1

2. The orbifold 3

3. D-branes on the orbifold 4

4. The moduli space of a regular D-brane 6

5. Spectrum of chiral operators 9

6. Supergravity duals 10

6.1 Massless Twisted States and Monodromy 10

6.2 Torsion Classes in Homology 12

7. New $N=1$ Dualities 13

8. Conclusions 14

A. Supergravity spectrum of chiral operators $\quad 15$

A.1 Twisted States of the $\mathbb{C}^{2} / \mathbb{Z}_{2}$ Orbifold 16

$\begin{array}{lll}\text { A.2 The Background } & 16\end{array}$

$\begin{array}{lll}\text { A.3 Mixing and Masses } & 17\end{array}$

$\begin{array}{ll}\text { B. Conventions for spin fields } & 18\end{array}$

\section{Introduction}

Orbifold singularities [1, 2] have been the object of much attention in string theory, as they provide solvable examples of conformal field theories and correspond to degenerate limits of Calabi-Yau families of vacua. Early on it was noticed that on calculating the spectrum of an orbifold there is an inherent ambiguity in the phases chosen for the twisted sector.[3] This ambiguity, discrete torsion, is classified by the group $H^{2}(\Gamma, U(1))$, where $\Gamma$ is the orbifold group.

Discrete torsion has been analyzed in the context of deformation theory in $[4$,

5] where it was noticed that discrete torsion singularities cannot be geometrically resolved, and that they provide totally distinct branches for compactifications of 
string theory. As a parameter, discrete torsion can be naturally associated to the NSNS 2-form in string theory, and thus it is natural to interpret it in terms of gerbes.[6]

More recently, it has been shown[7, 8] that discrete torsion can be analyzed in terms of D-branes at the orbifold by taking into account projective representations of the orbifold group. This is also needed for Matrix theory setups, as in [9]. In this way one builds quiver theories [10] for the modified orbifold which have a totally different content than the theories without discrete torsion. This analysis has been extended to the boundary state formalism in [11].

A new tool which to date has not been exploited to analyze this situation is to make use of the AdS/CFT correspondence [12]. In particular, the large $N$ limit of D3 branes at an orbifold was discussed in [13], but discrete torsion not considered. In this paper we analyze orbifolds with discrete torsion within the AdS/CFT correspondence.

The first task is to describe the physics of a particular type of orbifold with $\Gamma=\mathbb{Z}_{m} \times \mathbb{Z}_{n}$. We find the low energy field theory decsription of D3-branes at the singularity with discrete torsion for all possible choices of discrete torsion. This is done in Sections 2,3. The theories obtained are supersymmetric four-dimensional field theories of the brane-box type, with a modified superpotential. The orbifold can be recovered by the moduli space of vacua, which depends on the superpotential. This calculation is done in Section 4. We finish the field theory study in Section 5 where we compute the spectrum of chiral primaries of the CFT at the origin.

Secondly, in Section 6 we study the large $N$ theories associated to the orbifold with discrete torsion. The first fact that we need to consider is that they lie in the moduli space of deformations of more standard quiver diagrams, as can be argued along the lines of [14]. By following the deformations in supergravity, we are able to find a duality of type IIB string theories at weak coupling compactified on very different spaces. This is an example of mirror symmetry for RR backgrounds.

Subgroups of $\Gamma$ leave 2-planes fixed, which descend to fixed circles in the large $N$ limit. By a careful analysis of the string theory at the orbifold one can find the correct boundary conditions for the corresponding twisted sector states. These states are massless and hence survive in the supergravity limit. Thus discrete torsion is encoded in the near-horizon geometry, through different choices of boundary conditions for twisted fields. One can compare these results with the field theory from Section 5, and agreement is found. As a by-product we obtain geometric information on the orbifold with discrete torsion.

The analysis shows that if one considers resolutions by blowups of the classical geometry, on going around the fixed circles they are affected by monodromy. The monodromy encodes the discrete torsion, and shows that the topology of the $S^{5} / \Gamma$ is very different for the cases with and without discrete torsion. In particular, with a non-trivial monodromy one finds torsion classes in the homology, and then the 
discrete torsion parameter is the B-field flux around this homology two-cycle. This difference in topology also explains why the deformation theory of the orbifolds with and without discrete torsion are so different.

Section 7 deals with new dualities for large $N$ field theories. Two theories with the same field content at different values of marginal couplings have the same moduli space of flat directions and the same spectrum of chiral primary operators, and, furthermore, the coupling that distinguishes them does not appear in planar diagrams. The duality acts discontinuously on the space of couplings by a permutation of the roots of unity.

\section{The orbifold}

We analyze orbifolds of the Type IIB superstring of the form $\mathbb{C}^{3} / \Gamma$ with $\Gamma=\mathbb{Z}_{m} \times \mathbb{Z}_{n}$, and whose generators act on the coordinates by

$$
\begin{aligned}
& e_{1}:\left(z_{1}, z_{2}, z_{3}\right) \rightarrow\left(\alpha z_{1}, \alpha^{-1} z_{2}, z_{3}\right) \\
& e_{2}:\left(z_{1}, z_{2}, z_{3}\right) \rightarrow\left(z_{1}, \beta z_{2}, \beta^{-1} z_{3}\right)
\end{aligned}
$$

with $^{1} \alpha=\omega_{m}$ and $\beta=\omega_{n}$.

Because of the choice of action of the orbifold, we preserve $N=2$ supersymmetry in four dimensions, which can be broken to $N=1$ by the addition of D-branes at the singularity.

The discrete torsion parameter, is a 'bilinear' element $\epsilon \in H_{2}(\Gamma, U(1))$ such that

$$
\begin{aligned}
\epsilon\left(g_{1}, g_{2} g_{3}\right) & =\epsilon\left(g_{1}, g_{2}\right) \epsilon\left(g_{1}, g_{3}\right) \\
\epsilon\left(g_{1}, g_{2}\right) & =\bar{\epsilon}\left(g_{2}, g_{1}\right) \\
\epsilon\left(g_{1}, g_{1}\right) & =1
\end{aligned}
$$

for $g_{1}, g_{2}, g_{3} \in \Gamma$. As $\Gamma$ has two generators, $\epsilon$ is completely determined by the number

$$
\eta=\epsilon\left(e_{1}, e_{2}\right)
$$

It is easy to see that the solution of these equations is as follows. If $m, n$ are relatively prime, there is no discrete torsion. If we denote $p=\operatorname{gcd}(m, n)$, then we find $H_{2}(\Gamma, U(1))=\mathbb{Z}_{p}$. This includes the oft-studied special case $m=n$, where clearly $p=n$. The discrete torsion is thus determined by $\eta=\omega_{p}^{r}$, where $r=0,1, \ldots, p-1$. Let $s$ be the smallest non-zero number such that $\eta^{s}=1$. It is the integer $s$ which determines the physics, and for each value of $s$ the orbifolds will behave differently; for example, they will have a different resolution of singularities.

For later use, it will be useful to define a common root of unity $\lambda$, such that $\lambda^{m n}=1$. This phase will appear in the superpotential of the low energy gauge theory. It will also be useful to define $q=\operatorname{lcm}(m, n)$, the least common multiple of $m, n$.

\footnotetext{
${ }^{1}$ Throughout, we use the notation $\omega_{k} \equiv e^{2 \pi i / k}$.
} 


\section{D-branes on the orbifold}

We want to locate D3-branes at the orbifold. The construction for the low energy effective action follows $[10,7]$. In order to incorporate the discrete torsion parameter into the field theory, one has to consider all possible projective representations of the orbifold group with the same cocycle. That is, one considers representations where

$$
\gamma\left(e_{1}\right) \gamma\left(e_{2}\right)=\eta \gamma\left(e_{1} e_{2}\right)
$$

It is easy to see that given a projective representation $R$ of the group $\Gamma$ and a nonprojective representation $\chi$, the representation $R \otimes \chi$ is a projective representation of $\Gamma$ with the same discrete torsion parameter as $R$. By tensoring $R$ with all possible representations of $\Gamma$ we can obtain all possible projective representations of $\Gamma$.

We can thus build the analog of the regular representation of $\Gamma$ by considering $R=\oplus_{i} \operatorname{dim}\left(R_{i}\right) R_{i}$, with $R_{i}$ all the unitarily inequivalent projective representations of $\Gamma$, and out of the regular representation we should obtain the quiver diagram representing a D-brane which is outside the singularity. Any other D-brane configuration can be obtained by choosing the representation to be non-regular [15, 16]. String consistency imposes anomaly cancellation on the configuration.

One irreducible projective representation of $\Gamma$ is given in terms of $s \times s$ matrices

$$
\gamma\left(e_{1}\right)=\operatorname{diag}\left(1, \eta^{-2}, \eta^{-4}, \cdots, \eta^{-2(s-1)}\right), \quad \gamma\left(e_{2}\right)=\left(\begin{array}{ccccc}
0 & 1 & 0 & \cdots & 0 \\
0 & 0 & 1 & \cdots & 0 \\
\vdots & \vdots & \vdots & \ddots & \vdots \\
1 & 0 & 0 & \cdots & 0
\end{array}\right)
$$

Any other irreducible representation is given by multiplying $\gamma\left(e_{1}\right)$ by an $m^{\text {th }}$ root of unity $\alpha^{k}$ and $\gamma\left(e_{2}\right)$ by an $n^{\text {th }}$ root of unity $\beta^{\ell}$. This is equivalent to multiplying the representation $R_{i}$ by a character of the orbifold group, and thus producing another projective representation of the group, which is also irreducible. Two representations labeled by $(k, \ell)$ and $\left(k^{\prime}, \ell^{\prime}\right)$ are equivalent if $k=k^{\prime} \bmod m / s$ and $\ell=\ell^{\prime} \bmod n / s$, as can be seen by decomposing the representation into either characters of $\mathbb{Z}_{m}$ or $\mathbb{Z}_{n}$.

As all the irreducible projective representations are $s$-dimensional, we obtain the regular representation by taking all possible different projective representations with the same cocycle, multiplied by the dimension of the representation.

$$
R=\oplus_{k<m / s, \ell<n / s} s R_{k \ell}
$$

The total dimension of $R$ is $m n$, which is the order of the group, $|\Gamma|$.

The gauge theory living on the D-branes is given by a quiver diagram with $\frac{m}{s} \times \frac{n}{s}$ nodes, one for each representation. This theory is dual to an elliptic brane-box, as in $[17,18]$, a part of which appears in Figure 1. 
The nodes indicate the different $(k, \ell)$ gauge groups corresponding to each irreducible representation. To each of them we associate the gauge group $U\left(N_{k \ell}\right)$. For the regular representation, all of the gauge groups have equal rank, in particular $N_{k \ell}=s$. The arrows represent chiral multiplets transforming in the $(N, \bar{N})$ of the groups it connects. The nodes are connected by multiplications by specific characters of $\Gamma$.

Let us refer to the chiral multiplets corresponding to the horizontal lines as $\phi_{1 k \ell}$, the vertical ones as $\phi_{3 k \ell}$ and

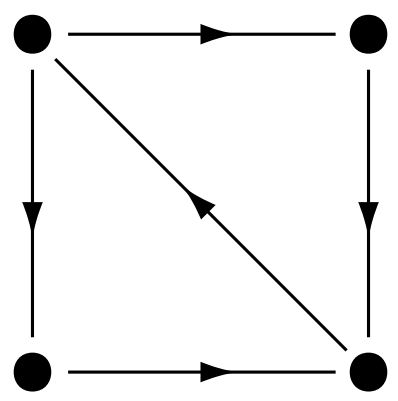

Figure 1: Part of the brane-box quiver. the diagonal ones as $\phi_{2 k \ell}$. To fully specify the theory, one also needs to give the superpotential. As brane box diagrams dual to theories with discrete torsion and without discrete torsion coincide, they can only differ in the superpotential [7].

The superpotential is of the form

$$
\sqrt{2} g \operatorname{tr}\left(\phi_{1} \phi_{2} \phi_{3}-\lambda^{s} \phi_{1} \phi_{3} \phi_{2}\right)
$$

A cubic term appears for each small triangle in the quiver, with a phase difference, depending on the orientation of the given triangle. Notice that it is the phase $\lambda$ that appears in the superpotential, instead of $\eta$. To see that this is correct, consider rephasing all of the chiral fields by arbitrary phases. This transformation would induce superpotential terms with a set of couplings $\lambda_{C}\left(\lambda_{A}\right)$ for (anti-)clockwise triangles.

$$
\sqrt{2} g \operatorname{tr}\left(\lambda_{A} \phi_{1} \phi_{2} \phi_{3}-\lambda_{C} \phi_{1} \phi_{3} \phi_{2}\right)
$$

There is one combination of couplings which is invariant under field redefinitions, and hence cannot be eliminated. It is the quotient

$$
\frac{\prod_{\Delta_{C}} \lambda_{C}}{\prod_{\Delta_{A}} \lambda_{A}}=\eta
$$

and turns out to equal the discrete torsion. In this way, the discrete torsion is encoded in the theory. With our choice of conventions, $\lambda_{C}=\lambda^{s}, \lambda_{A}=1$. We have chosen the couplings so that they all are the same, and therefore the quantum symmetry is easier to construct. We could just as well have made all the couplings equal except at one triangle, where we would insert the phase $\eta$. Notice that when $m=n$ with only one node in the diagram, this reduces to the case considered in $[7,8]$.

It should be possible to deduce the form of the superpotential directly through string computations. In particular, we wish to show that the diagrams in Figure 2 differ essentially in phase. 
In the field theory, there is a vacuum diagram which encodes the phase $\eta$, and can thus be traced back to the superpotential (3.4). It uses each of the cubic vertices exactly once, and thus is proportional, in field theory, to the ratio of phases (3.6) (or its inverse, depending on chirality). When drawn on top of the quiver, it has the topology of a net of hexagons (one for each node), as shown in Figure 3 .

In string theory, we would evaluate this using projected fields $\phi_{a, i n v}=\sum_{g \in \Gamma} r_{a}(g) \gamma^{-1}(g) \phi_{a} \gamma(g)$. The diagram is equivalent to a genus one worldsheet with $\mathrm{mn} / \mathrm{s}^{2}$ holes (one for each node in the quiver). Thus the result is a product of traces, one for each node labelled $i, j$, each of the form

$\operatorname{tr}_{R_{i j}} \gamma^{-1}\left(g_{i j, 1}\right) \gamma\left(g_{i j, 2}\right) \gamma^{-1}\left(g_{i j, 3}\right) \gamma\left(g_{i j, 4}\right) \gamma^{-1}\left(g_{i j, 5}\right) \gamma\left(g_{i j, 6}\right)$
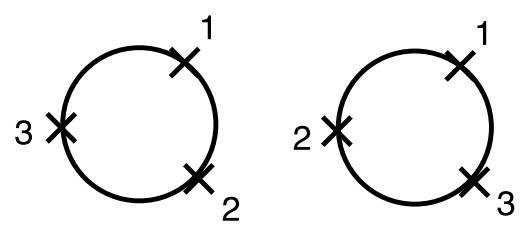

Figure 2: The two disc diagrams.

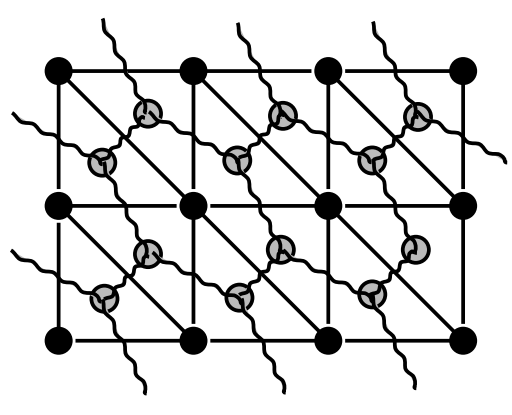

Figure 3: A portion of the dual where the group elements are paired, viz $g_{i, j, 3}=$ hexagon Feynman diagram.

$g_{i+1, j, 6}^{-1}, \quad g_{i, j, 4}=g_{i+1, j+1,1}^{-1}, \quad g_{i, j, 5}=g_{i, j+1,2}^{-1}$. This result is the analogue of the single node computation of Ref. [7].

It should be possible to reduce the product of traces by implementing the relation (3.1) and using character formulas. Furthermore, the results given later in the paper are fully consistent with the form of the superpotential, (3.4).

\section{The moduli space of a regular D-brane}

In order to verify that we have the correct field theory, we should consider the moduli space of vacua for a regular D-brane. In particular, one should have a three dimensional component where the D-brane lives away from the singularities, which corresponds to the total orbifold space minus the fixed lines. When the D-brane approaches one of the fixed lines, or the fixed point, the D-brane can fractionate, and the dimension of the moduli space jumps.

From the orbifold point of view, the moduli space is described by six variables with three relations. Only the $\Gamma$-invariant polynomials in the variables are good functions on the space. This polynomial ring is generated by $x=z_{1}^{m}, y=z_{3}^{n}, z=z_{2}^{q}$, $u=\left(z_{1} z_{2}\right)^{n}, v=\left(z_{2} z_{3}\right)^{m}$ and $w=z_{1} z_{2} z_{3}$, where $q$ is the least common multiple of 
$m, n$. There are three relations ${ }^{2}$

$$
\begin{aligned}
& f_{1}=x^{q / m} y^{q / n} z-w^{q}=0 \\
& f_{2}=v x-w^{m}=0 \\
& f_{3}=u y-w^{n}=0
\end{aligned}
$$

and we have three complex lines of singularities where two out of the three $x, y, z$ are equal to zero. These are the lines which are fixed by some elements of the orbifold.

From the gauge theory, we need to construct the equivalent of variables $x, y$, $z, u, v, w$ in terms of gauge invariant polynomials of the chiral fields. As is well known from Matrix theory [19], one should hope to replace the equations (4.1)-(4.3) by matrix versions. If the matrices are made to commute, then we can identify variables $x, y, z, u, v, w$ for individual branes by their eigenvalues. In particular, if we want branes in the bulk, we should require that $x, y, z$ be all invertible (in this case as matrices).

Referring to the quiver, the natural gauge invariant coordinates are given by traces of closed loops in the diagram. Matrix coordinates will be given by taking the same closed loops without the trace, as they represent matrices whose eigenvalues are gauge invariant quantities, since gauge transformations act by similarity transformations.

Let us consider the variables $\tilde{x}_{j}=\prod_{i=1}^{m / s} \phi_{1 i j}$, and $\tilde{y}_{j}=\prod_{i=1}^{n / s} \phi_{3 j i}$, and similarly $\tilde{z}_{j}$ along diagonals. It is easy to see that all the $\tilde{x}_{i}$ are isospectral up to phases, the same being true for all the $\tilde{y}_{j}$, under the assumption that all the chiral matrices that appear in the diagram are invertible and satisfy the $F$-constraints. The proof goes by making use of moves which relate different matrices, and by application of the Cayley-Hamilton theorem. An example of a move is shown in Figure 4.

The simple moves are given by solving the $F$ constraints for a single chiral multiplet. Under such a move, we get a phase given by the ratio of the two associated triangle couplings in the superpotential.

The eigenvalues of the matrix $\tilde{x}_{j}$ are determined by the roots of the characteristic polynomial of $\tilde{x}_{j}$.

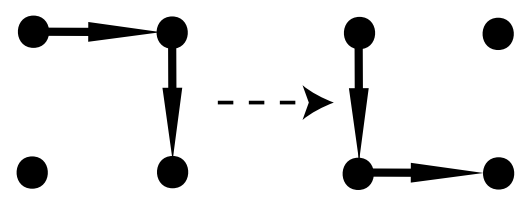

Figure 4: Allowed moves As $\tilde{x}_{j}$ satisfies its own characteristic polynomial, we have an identity $P\left(\tilde{x}_{j}\right)=0$, which we can use as a matrix equation. We can now change a row by multiplying by $\phi_{2}$ at the end. As we want a matrix equation that begins and ends on the same node, we can complete it by multiplication by the rest of the triangle that returns the operator to the starting node. We can now make use of the moves to shift the polynomial equation one step down, as shown in the Figure 5.

Indeed, apart from a global phase in the roots, the two characteristic polynomials are the same, and therefore the two matrices have the same content. If we multiply by the triangle operator and use the $F$-term constraints, we can move the polynomial

\footnotetext{
${ }^{2}$ Note that when $m=n=q$, the variables $u, v$ are redundant, and the system reduces to just $f_{1}=0$.
} 
equation along the rows, and hence all of the $\tilde{x}_{j}$ have the same roots (modulo phases), and therefore they are equivalent when considered as matrix variables. Similarly for all the $\tilde{y}_{k}$ and $\tilde{z}_{k}$. As a result, we need only consider the matrices $\tilde{x}_{1}, \tilde{y}_{1}, \tilde{z}_{1}$.

Now, using the moves, we can also see using (3.6) that

$$
\tilde{x}_{1} \tilde{y}_{1}=\eta \tilde{y}_{1} \tilde{x}_{1}
$$

This means that if $\tilde{y}_{1}$ is invertible, then the eigenvalues of $\tilde{x}_{1}$ are only well defined modulo $\eta$, and hence the trace of $\tilde{x}_{1}$ vanishes, so $\tilde{x}_{1}$ alone is not a good matrix variable to describe the moduli space. On the other hand, it shows us also that the matrices must be of order $s \times s$ in order to find a represen-

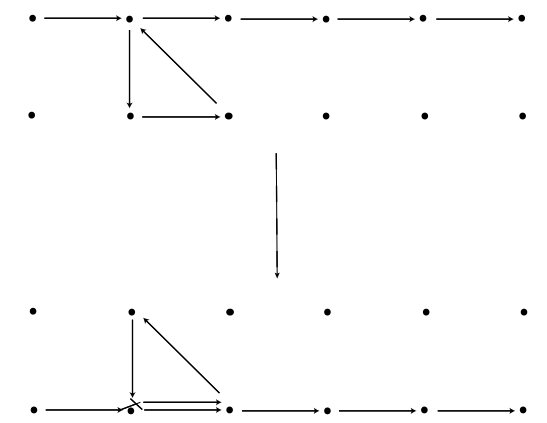

Figure 5: Relating the rows. tation of the algebraic equation (4.4). This is exactly the size of the matrices in the regular representation of the quiver.

Now we can take $x=\tilde{x}_{1}^{s}$, and similarly $y=\tilde{y}_{1}^{s}$ and $z=\tilde{z}_{1}^{s}$. These matrices will commute amongst themselves, and they have $s$ identical eigenvalues. The matrix $w$ is constructed from any small triangle in the graph, and it is easy to see that $w$ commutes with all $x, y, z$. $w$ is defined up to a phase depending only on the orientation of the triangle.

One can then see that the matrices $x, y, z, w$ satisfy eq. (4.1) by making use of the $F$-constraint moves. As the matrices $x, y, z, w$ are all $s \times s$ and proportional to the identity, we can identify the eigenvalues with the coordinates $x, y, z, w$ of the algebraic variety which describes the orbifold. The two different choices of $w$ differ by a root of unity which cancels in the equation, as the order of $\lambda^{s}$ divides $q$. Similar analyses can be used to construct the constraints (4.2)-(4.3), using the zigzags $u_{j}=\prod_{i=1}^{n / s}\left(\left(\phi_{1} \phi_{2}\right)_{i j}\right)^{s}$ and $v_{i}=\prod_{j=1}^{m / s}\left(\left(\phi_{2} \phi_{3}\right)_{i j}\right)^{s}$.

For a total number of branes which is $N$ times that of the regular representation, we get mutually diagonalizable matrices whose eigenvalues have a common multiplicity of $s$ each. Hence, the spectrum of regular D3 branes gives a moduli space which is a symmetric product of the moduli space of a single regular brane. Namely

$$
\mathcal{M}_{N}=\mathcal{M}_{1}^{N} / S_{N}
$$

Notice that if we relax the invertibility of the matrices, then the branes can only fractionate at the fixed complex lines, as we need two out of the three matrices $x, y, z$ to be non-invertible in order for the previous arguments to fail, which is just as expected.

Consider now adding fractional D3 branes. The configuration is allowed only if the gauge theory anomaly vanishes, that is, if the fractional D-brane is constructed by combinations of effective $N=2$ directions [20]. The effective $N=2$ directions are 
associated with the fixed complex lines of subgroups of $\Gamma$, on which fractional branes can reside because the orbifold singularity is not isolated. In principle, one can see a brane moving in the Coulomb branch only if it is a linear combination of $N=2$ effective directions, and thus is automatically anomaly free. This will be important when we compare with the supergravity analysis.

\section{Spectrum of chiral operators}

In order to make the comparison with supergravity later on, we need to calculate the spectrum of single trace chiral primaries [12, 21, 22].

The quantum symmetry generators $Q_{1}$, and $Q_{2}$, which correspond to the group of characters of $\Gamma$ act by

$$
\begin{aligned}
Q_{1}: \phi_{1, i, j} & \rightarrow \alpha \phi_{1, i+1, j} \\
\phi_{2, i, j} & \rightarrow \alpha^{-1} \phi_{2, i+1, j} \\
\phi_{3, i, j} & \rightarrow \phi_{3, i+1, j} \\
Q_{2}: \phi_{1, i, j} & \rightarrow \phi_{1, i, j+1} \\
\phi_{2, i, j} & \rightarrow \beta \phi_{2, i, j+1} \\
\phi_{3, i, j} & \rightarrow \beta^{-1} \phi_{3, i, j+1}
\end{aligned}
$$

and they only act by shifts on the gauge fields. It is obvious that they are invariances of the field theory as long as all gauge couplings and Yukawa couplings are equal, which is the orbifold point. This identification of the quantum symmetry is necessary in order to define the twist charge of operators in the field theory. In particular, untwisted operators correspond to quantum symmetry singlets. The field theory also has a $U(1)^{3}$ symmetry. One of the $U(1)^{\text {'s }}$ is the $R$-symmetry and the other $U(1)$ 's twist the field $\phi_{1}, \phi_{2}, \phi_{3}$ by global phases.

Untwisted single-trace chiral primary operators are given schematically by

$$
O_{a b c}=\operatorname{tr} \phi_{1}^{a} \phi_{2}^{b} \phi_{3}^{c}
$$

for any closed loop in the quiver. In order for the operator to be a conformal primary, it must be such that dragging one field around and using the cyclicity of the trace induces no phase. The true operator is a linear combination of all of the possible terms with the same structure that are related by the $F$ constraints. The other linear combinations will correspond to chiral fields which are not primary.

For a primary to be untwisted, it must satisfy a more stringent constraint. It is straightforward to show that untwisted primaries may be written as

$$
\operatorname{tr} P\left(\phi_{1}^{m}, \phi_{2}^{q}, \phi_{3}^{n}, \phi_{1} \phi_{2} \phi_{3},\left(\phi_{1} \phi_{2}\right)^{n},\left(\phi_{2} \phi_{3}\right)^{m}\right)
$$


where $P$ is any monomial. The point is that the variables in $P$ all transform without phase under the quantum symmetry and commute with one another, so that they are not constrained by the presence of discrete torsion. These monomials are in oneto-one correspondence with monomials in $x, y, z, u, v, w$ of the last section. These operators are in fact superconformal primaries, and additional conformal primaries may be constructed by inserting factors of $W_{\alpha}^{2}$.

Twisted states come in families of the form

$$
\mathcal{O}_{1}^{a}=\operatorname{tr} \phi_{1}^{a m / s}
$$

for some integer $a$, in multiplicity $n / s$. Similar twisted operators occur in $\phi_{2}$ and $\phi_{3}$ as well. These are operators where one closes loops horizontally, vertically or diagonally. It is a non-trivial fact that these operators exhaust the list of twisted superconformal primaries. Note that the operator with $a$ a multiple of $s$ is untwisted.

\section{Supergravity duals}

\subsection{Massless Twisted States and Monodromy}

The supergravity duals of the previous D-brane configurations are obtained by looking at the near-horizon geometry of the D3-branes at the orbifold, and they will correspond to geometries of the type $A d S_{5} \times S^{5} / \Gamma$, where $\Gamma$ acts by isometries of the $S^{5}$. Our first task is to understand how the orbifold encodes the discrete torsion.

Because of our choice of group action, some orbifold elements will leave invariant three fixed circles of the $S_{5}$, which descend from the fixed planes of the orbifold. Along these circles, we have locally a singularity of the type $\mathbb{C}^{2} / \mathbb{Z}_{k}$, where the $\mathbb{Z}_{k}$ is abelian and generated by the element that fixes the point, that is, we will have a fibration of the $A_{k-1}$ singularities along these circles, which can be locally resolved by $k-1$ blowup parameters into a bouquet of $k$ spheres which intersect according to the extended Dynkin diagram of the $A_{k-1}$ group with one relation among these cocycles.

The twisted states are associated to elements of the group $\Gamma$. They can survive in the supergravity limit if they correpond to massless particles. It is known that zero modes for blowup modes are not present in sufficient numbers to smooth out the geometry, so it is not possible to study these models within classical geometry; we must turn to string theory for detailed computations. Massless twisted states have support at the fixed points of the group element they are associated with. Thus at each fixed circle we have massless states in the string theory which survive the low energy limit, and are associated with the group elements that fix the circle.

By going around the fixed circle on a closed loop, we are actually performing a twist by the group elements which don't fix the circle, because the fixed circle is an 
invariant subspace of the other elements of the orbifold group. The circle is of radius $k^{-1}$.

For the twisted strings that live at the orbifold circles, going around the loop one picks up a phase equal to the discrete torsion of the cycle acting on the group element to which the twisted state corresponds. This is the discrete torsion phase in the partition function. In our case, it sets the boundary conditions for the massless twisted sector states.

Consider for example the singularity of $z_{2}=z_{3}=0$ and the $g=e_{2}^{\ell}$ twisted sector corresponding to the subgroup which fixes the circle. When we set the boundary conditions for the twisted field, we get

$$
\mathcal{O}_{g}(t+2 \pi h R / m)=\epsilon\left(g, e_{1}^{h}\right) \mathcal{O}_{g}(t)
$$

for $t$ the geodesic coordinate along this circle measured in units of $R$, the radius of the AdS space. This circle is of size $m^{-1}$, and we wind around it $h$ times. Winding once around is the same as twisting by $e_{2}$ in the unorbifolded covering space.

The blowup spheres are related to the discrete Fourier transform of the twisted states, and the action of the discrete torsion phase (which one can associate to the quantum symmetry of the local orbifold) becomes an automorphism of the Dynkin diagram which preserves the intersection form and the orientation. Thus the geometry differs from the standard $S^{5} / \Gamma$ space in that the singularities have monodromy of the resolving spheres. This is a special example of monodromies that have been studied in [23, 16], which are related to canonical singularities [24].

The equation (6.1) then becomes for the fields on the basis of the spheres

$$
\hat{\mathcal{O}}_{i}(t+2 \pi h R / m)=\hat{\mathcal{O}}_{i+a(m / s) h}(t)
$$

The precise discrete torsion value is encoded in the possible integer values of $a$, which are relatively prime to $s$. Let us take $h=1=a$ for convenience. Then on going around the fixed circle the monodromy acts as shown in Figure 6.

It is worth pointing out that although we start with $k-1$ twisted sectors, we have a bouquet of $k$ shrunken spheres. The relation in homology makes one linear combination of the local fields untwisted, and thus does not belong to the twisted string states. The counting of twisted states is the same in both approaches.

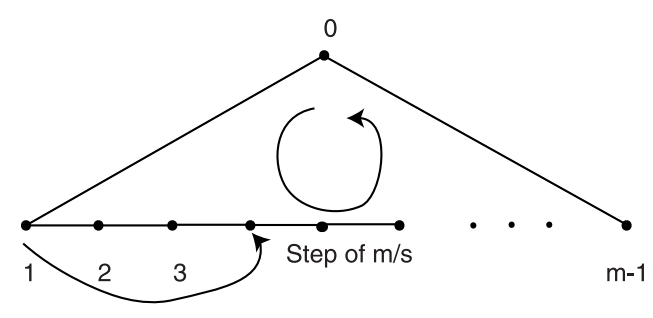

Figure 6: Monodromy of spheres

Details of the exact calculation of the spectrum of masses are given in the Appendix A. The masses are given exactly by the tree level mixing induced by the RR and NSNS backgrounds. It is not difficult to see that the spectrum of masses of chiral twisted state operators gives rise to conformal fields of the appropriate dimensions 
and twist charge as the ones predicted by the orbifold low energy effective field theory. In fact, one can see that the monodromy of the spheres makes the fields periodic with period $2 \pi R s / m$, as opposed to $2 \pi R / m$, so the circles look $s$ times larger than the geometric circle, and the quantization of the masses of the states gives a factor in the mass which is proportional to $m s^{-1} R^{-1}$ as opposed to the factor of $m R^{-1}$ coming just from the size of the circle (c.f. eq. (5.9)). This is exactly the spectrum of states predicted by the field theory too.

Notice also that the value of $a$ is irrelevant for this calculation, as it amounts to a different permutation of the spheres when going around the singularity. This is also in accord with the field theory. This is the first check on the duality.

We should also point out that the spectrum of D-branes that can leave the singularity is also equal in both theories (supergravity and field theory), as any fractional D-brane that is seen in the bulk of supergravity is either untwisted, or consists of a collection of D-brane states that are fixed at the orbifold circles.

\subsection{Torsion Classes in Homology}

Notice that the singular manifold thus described has torsion on homology two-cycles. Upon monodromy on the circle, different spheres are identified.

On the singularity that we have just studied, there are $\mathrm{m} / \mathrm{s}$ equivalence classes in homology at the singularity. They can be represented as the average cycles

$$
\tilde{C}_{i}=\frac{1}{s}\left[C_{i}+C_{i+m / s}+C_{i+2 m / s}+\ldots\right]
$$

and they intersect according to the $A_{m / s-1}$ Dynkin diagrm.

The relation in homology is given by

$$
\sum_{i} C_{i}=0=s \sum_{i} \tilde{C}_{i}
$$

so calling $T=\sum_{i} \tilde{C}_{i}$ we can prove that $s T=0$, but $T \neq 0$ as a homology cycle. Indeed, if we wrap a brane around the $T$ cycle, the brane is locally made of $\mathrm{m} / \mathrm{s}$ spheres, and is not allowed to leave the circle of singularities, since it can be made to correspond to a root of the $A_{m-1}$ lattice, and therefore it can not be deformed away from the singular cycle. This argument works for $t$ copies of the cycle for all $t<s$. Thus the torsion cycle is of order $s$.

This is seen also in the field theory, because one cannot solve the $F$ constraints for $\phi_{1}, \phi_{2}, \phi_{3}$ all different from zero, unless one has rank $s$ on each of the gauge groups in the quiver.

This establishes a direct correspondence between fractional regular branes and the torsion classes of the manifold. The existence of a torsion class is consistent with the absence of blowup modes in the string spectrum. As the fractional $D p$ branes also carry an anomalous $D(p-2)$ brane charge [25] (because the orbifold has constant 
B field along each of the blowup cycles), one can have a BPS torsion class with finite tension.

\section{New $N=1$ Dualities}

Notice that the quivers we have obtained are identical to quivers of standard type without discrete torsion. They only differ in the superpotential, and in fact, they lie in the moduli space of deformations of the standard quiver theories [14]. For a single-node quiver, the field theory is a marginal deformation of the $N=4$ theory

$$
W=\sqrt{2} g \operatorname{tr} \phi_{1}\left[\phi_{2}, \phi_{3}\right]-\sqrt{2} g\left(\lambda^{s}-1\right) \operatorname{tr} \phi_{1} \phi_{2} \phi_{3}
$$

Alternatively, this may be rewritten in terms of $f_{a b c}$ and $d_{a b c}$ as appears in Ref. [14]. In the case of multinode quiver theories, the superpotential is a marginal deformation of standard $N=1$ quiver theories.

Thus, with the AdS/CFT dictionary in hand, these field theory deformations act by geometrically deforming the theory which started with $S^{5}$, or $S^{5} / \Gamma$. In particular, we expect that the marginal deformation corresponds to replacing the 5 -sphere with a deformed version, and also turning on 2-form fields.

In supergravity it has been argued that one cannot observe some of these deformations [26]. However, as we deform the superpotential, the conditions for a field being primary change, as the moduli space of couplings is curved. In the field theory this is seen because conformal invariance imposes restrictions on the couplings.[14]

The moduli space of the theory changes when we follow these deformations. In particular, we have to find solutions of the $F$-constraints, which take the form

$$
\phi_{1} \phi_{2}=\omega \phi_{2} \phi_{1}
$$

For generic $\omega$, there is no solution to these matrix equations with $\phi_{1} \phi_{2} \neq 0$ at finite $N$, and therefore the classical moduli space disappears. But on the other hand, we can elevate the previous equation to non-commutative geometry as in [27], if we let

$\phi_{i}$ be operators acting on an infinite dimensional Hilbert space. In this case we would be forced onto the large $N$ field theory from the start.

As a marginal perturbation, $\lambda$ would seem to be a continuous parameter. However, properties of the theory change discontinuously as we change $\lambda$. There is a direct analogy here to the $\theta$-angle of six-dimensional gauge theories, [23] which are also related to non-commutative geometry.[28] For values of $\lambda$ which are roots of unity, the classical moduli space has solutions for finite matrices, and it is exactly in this case where we have the dual theory with the orbifold.

Thus, for each of these theories under the conditions for the Maldacena conjecture to be reduced to supergravity, we have two types of supergravity duals. Namely, $A d S_{5} \times \tilde{S}_{5}$ where we have a deformed sphere $\tilde{S}^{5}$ and $A d S_{5} \times S^{5} / \Gamma_{d}$, where we use 
the subscript $d$ to indicate the presence of discrete torsion. (This is the extreme case where the quiver theory has only one node, but one can restate it for the general case by writing the extra quotients properly.) These theories are mirror to one another, even at finite $N$.

At infinite $N$, there are additional field theory dualities relating different values of $\lambda$. On $A d S_{5} \times S^{5} / \Gamma_{d}$ we have various choices of discrete torsion parameter. But it can easily be seen that neither the chiral ring nor the classical moduli space depends on this choice, once $s$ is fixed. Moreover one sees that the discrete torsion parameter can only be measured in chiral correlation functions involving twist fields that have support at different singularities. In the supergravity approximation, in order for twist fields to interact, they should be able to be at the same spacetime point. Thus all of these amplitudes are suppressed because the twisted strings have to extend far away along the sphere in order to interact.

On the field theory side, these amplitudes vanish in the free field limit, as they would involve, for example, the OPE of $\operatorname{tr}\left(\phi_{1}^{k}\right) \operatorname{tr}\left(\phi_{2}^{k}\right) \operatorname{tr}\left(\phi_{3}^{k}\right)$. Notice that if the free field limit is the correct way to calculate three point functions (just as in $A d S_{5} \times S^{5}$ )[29] then the discrete torsion parameter disappears in the supergravity regime. Indeed, discrete torsion appears only in a non-planar diagram, as in Figure 3.

It would be natural then to identify all of these CFT theories as dual pairs. They meet all of the requirements of $N=1$ duality, as they have the same moduli space of flat directions and the same spectrum of chiral primaries. At large $N$ they also have the same set of three point correlation functions.

This duality would act by exchanging the fundamental roots of unity. It is not continous on the parameter $\lambda$, but it is a well known mathematical object: it is the Galois group of the algebraic extension $\mathbb{Q}(\eta)$. In our case this Galois group can also be made to act on the algebra generated by the matrices $x, y, z, u, v, w$ if all of their components are algebraic numbers, but the significance of this curiosity is at best obscure.

\section{Conclusions}

We have analyzed some Abelian orbifolds with discrete torsion from the D-brane perspective. Our results show that the simple cases give rise to deformations of known theories of the brane box type, which differ only in the superpotential. This result extends the work of $[7,8]$. We tested the field theory by reproducing the orbifold as the moduli space of D-branes and by calculating the spectrum of chiral primaries.

In analyzing the field theory with the AdS/CFT correspondence, we discovered a new geometric picture for the orbifolds with discrete torsion. Indeed we found that as the fixed planes of singularities of the orbifold become fixed circles in the AdS/CFT correpondence, the study of massless twisted states on these fixed circles revealed 
that the blowup modes of the singularities have monodromy. The resolved geometry then has a different topology than the standard orbifold, and provides a geometrical understanding of the discrete torsion. Also, the obstructions to resolving the CalabiYau singularities may be traced back to the existsence of monodromy of the blowup modes. We have explicitly checked this picture by calculating the spectrum of chiral primaries in the supergravity/string theory and comparing with the field theory.

The AdS/CFT correspondence can then be used to prove new examples of dual-

ity. Indeed, as the theories with discrete torsion are seen to be given by deformations of standard orbifolds, one can follow these deformations at the supergravity level. This provides new examples of mirror symmetry, as both string theory descriptions are weakly coupled.

In the large $N$ limit we have also found new dualities. These dualities appear because the spectrum of operators in the supergravity is insensitive to the discrete torsion parameter, and the three-point functions of chiral twisted states that could measure this parameter vanish in this limit. The duality acts discontinuosly on the parameters defining the theory by permuting the different roots of unity.

It would be interesting to get a better understanding of the deformation theory and resolution of singularities within the D-brane language along the lines of [30], and to extend these results for other marginal and relevant deformations of the field theory. We would also like to extend the discrete torsion models to other geometries, wuch as the conifold[31, 32, 33]. This is work in progress [34].

\section{Acknowledgments}

Partially supported by the United States Department of Energy, grant DE-FG0291ER40677 and an Outstanding Junior Investigator Award.

\section{A. Supergravity spectrum of chiral operators}

In this appendix we set up the calculation of the supergravity spectrum of states in the orbifolds with discrete torsion. Our construction is done following the background normal coordinate expansion for Ramond-Ramond background type of calculations explained in $[35,36]$. These results coincide with supergravity calculations obtained in [37] in cases where smooth geometry exists, but generalizes to spaces which have less local symmetry. For simplicity we will study the local $\mathbb{C}^{2} / \mathbb{Z}_{2}$ fixed circles (only because it is easier to write the twisted vertex operators). The results generalize immediately to any other orbifolds.

The analysis is completely standard. The spin field $S^{\alpha}$ is written in terms of $\operatorname{Spin}(5,1) \times \operatorname{Spin}(4)$ spin-fields: $S^{\alpha} \rightarrow\left\{S^{A} \Sigma^{a}, S_{A} \Sigma^{\dot{a}}\right\}$. Conventions for operator products may be found in the Appendix B. 


\section{A.1 Twisted States of the $\mathbb{C}^{2} / \mathbb{Z}_{2}$ Orbifold}

At the fixed circle, we have a $\mathbb{C}^{2} / \mathbb{Z}_{2}$ orbifold. The twisted sector massless strings are given by the following operators.

In the $N S N S$ sector, we find

$$
\mathcal{O}_{N S}^{a b} \sim \Sigma^{a} \tilde{\Sigma}^{b} e^{-\phi} e^{-\tilde{\phi}} \sigma_{6} \sigma_{7} \sigma_{8} \sigma_{9} e^{i k \cdot X}
$$

These transform as $(\mathbf{1},(\mathbf{1}+\mathbf{3}, \mathbf{1}))$ under $\operatorname{Spin}(5,1) \times \operatorname{Spin}(4)$. and correspond to the $N S \theta$-angle, $\Theta_{N S}$ and the triplet of blowup modes.

In the $R R$ sector, we find

$$
\mathcal{O}_{R, A B} \sim S_{A} \tilde{S}_{B} e^{-\phi / 2} e^{-\tilde{\phi} / 2} \sigma_{6} \sigma_{7} \sigma_{8} \sigma_{9} e^{i k \cdot X}
$$

which transform as $(\mathbf{6}+\mathbf{1 0},(\mathbf{1}, \mathbf{1}))$. The $\mathbf{6}$ is the field strength of the $R R \theta$-angle, $\partial_{\mu} \Theta_{R}$.

\section{A.2 The Background}

The background that we are interested in must be a singlet under both $\operatorname{Spin}(4)$ and $\operatorname{Sp}(2) \sim \operatorname{Spin}(5) \subset \operatorname{Spin}(5,1)$. The $R R$ part is then determined as follows. The $\mathbf{6}$ of $\operatorname{Spin}(5,1)$ has one $S p(2)$ singlet, while the $\mathbf{1 0}$ has none. We thus find that the $R R$ background vertex is

$$
V_{R R} \sim h \int\left(J_{A B} \epsilon_{a b} S^{A} \Sigma^{a} \tilde{S}^{B} \tilde{\Sigma}^{b}+\alpha J^{A B} \epsilon_{\dot{a} \dot{b}} S_{A} \Sigma^{\dot{a}} \tilde{S}_{B} \tilde{\Sigma}^{\dot{b}}\right) e^{-\phi / 2} e^{-\tilde{\phi} / 2}
$$

where $h$ is a normalization constant, $\alpha$ is a fixed phase and $J$ is the antisymmetric $S p(2)$ invariant.

First, let us note that two $R R$ background insertions give a log divergence when they are close together

$$
V_{R R}(z) V_{R R}\left(z^{\prime}\right) \sim 16 h^{2} \ln \varepsilon \int\left(\psi^{\mu} \tilde{\psi}_{\mu}-\psi^{M} \tilde{\psi}_{M}\right) e^{-\phi} e^{-\tilde{\phi}}
$$

The signature here is $(5,5)$, and this divergence corresponds to a contribution to the graviton $\beta$-function. The other contribution may be computed directly within the normal coordinate expansion, and takes the form

$$
\frac{1}{4} \log \varepsilon \int R_{i j} \psi^{i} \tilde{\psi}^{j} e^{-\phi} e^{-\tilde{\phi}}
$$

Thus we fix the normalization of the $R R$ background

$$
h R=\frac{1}{2 \sqrt{2}}
$$

where $R$ is the radius of the $A d S_{5}$ metric. 


\section{A.3 Mixing and Masses}

Here we compute the masses of twisted eigenstates. In the presence of the $R R$ background, there is operator mixing at lowest order. In particular, we find mixing between $\Theta_{N S}$ and $\Theta_{R}$, as follows. Using the notations (A.1),(A.2), these singlets have vertex operators of the form

$$
\begin{aligned}
\mathcal{O}_{N S}^{(1)} & \sim \Theta_{N S} \epsilon_{a b} \mathcal{O}_{N S}^{a b} \\
\mathcal{O}_{R}^{(1)} & \sim \partial_{\mu} \Theta_{R}\left(\Gamma^{\mu}\right)^{A B} \mathcal{O}_{R, A B}
\end{aligned}
$$

Mixing is induced through the Fischler-Susskind mechanism - there are logarithmic divergences between the background $V_{R R}$ and these operators.

Consider the equation of motion for the $R R$ singlet. We find mixing through

$$
\mathcal{O}_{N S}^{(1)}(z) \cdot V_{R R}\left(z^{\prime}\right) \sim 2 h \Theta_{N S} J_{A B} \not k^{A C} \not k^{B D} \log \varepsilon \mathcal{O}_{R, C D}\left(z^{\prime}\right) .
$$

Thus we find an equation of motion

$$
\frac{1}{2} k^{2} \partial_{\mu} \Theta_{R}\left(\Gamma^{\mu}\right)^{C D}+2 h J_{A B} \not k^{A C} \not k^{B D} \Theta_{N S}=0
$$

and thus mass terms

$$
\frac{n^{2}}{R^{2}} \Theta_{R}+4 i h \frac{n}{R} \Theta_{N S}
$$

Similarly, mixing for the NSNS singlet is induced through

$$
\mathcal{O}_{R}^{(1)}(z) \cdot V_{R R}\left(z^{\prime}\right) \sim h \partial_{\mu} \Theta_{R} J_{A B}\left(\Gamma^{\mu}\right)^{A B} \log \varepsilon \epsilon_{a b} \mathcal{O}_{N S}^{a b}\left(z^{\prime}\right)
$$

Putting these results together, we have a mass matrix

$$
\left[m^{2}\right]=\left(\begin{array}{cc}
\left(\frac{k}{R}\right)^{2} & -8 i h\left(\frac{k}{R}\right) \\
+4 i h\left(\frac{k}{R}\right) & \left(\frac{k}{R}\right)^{2}
\end{array}\right)
$$

where $k$ is the momentum along the fixed circle in units of $R$.

Within the AdS-CFT correspondence[12, 21, 22], we expect that the eigenvalue masses correspond to the dimensions of operators in the boundary CFT:

$$
\Delta=2+\sqrt{4+m^{2} R^{2}}
$$

Given the result A.13, we find a tower of states

$$
\Delta_{1}=2+|k \pm 2|
$$

where $k \in \mathbb{Z}$.

Similiarly, one obtains for the triplets

$$
\Delta_{3}=2+|k|
$$


The interesting part of this calculation comes from the origin of the mass shift, which can be traced to the $\sigma$-model coupling proportional to $R_{a b c d} \psi^{a} \psi^{b} \bar{\psi}^{c} \bar{\psi}^{d}$, and thus for spaces where $R$ is not symmetric one should be able to observe the effect here.

It is clear that these calculations are independent of the fact that we had a $\mathbb{C}^{2} / \mathbb{Z}_{2}$ orbifold. The structure of states is the same for any orbifold group. The different orbifolds $\mathbb{C}^{2} / \mathbb{Z}_{n}$ will have instead $n-1$ twisted sectors.

The singlet states are chiral. Because of the monodromy of the circle $k=\mathrm{am} / \mathrm{s}$,

and the dimensions of the operators match the $\operatorname{tr}\left(\phi_{i}^{(a m / s)}\right)$, with multiplicities. The other operators are related to $\operatorname{tr}\left(W^{2} \phi_{i}^{(a m / s)}\right)$.

\section{B. Conventions for spin fields}

Under $\operatorname{Spin}(5,1) \times \operatorname{Spin}(4) \subset \operatorname{Spin}(9,1)$, the spin fields decompose as $S^{\alpha} \rightarrow\left\{S^{A} \Sigma^{a}, S_{A} \Sigma^{\dot{a}}\right\}$. Here $A$ is an $\operatorname{Spin}(5,1)$ spinor index and $a, \dot{a}$ are $\operatorname{Spin}(4)=S U(2) \times S U(2)$ indices. We denote vector indices by $\mu, \nu, \ldots$ for $\operatorname{Spin}(5,1)$ and $M, N, \ldots$ for $\operatorname{Spin}(4)$.

Operator products are

$$
\begin{gathered}
\Sigma^{a}(z) \Sigma^{b}(0) \sim \frac{\epsilon^{a b}}{z^{1 / 2}} \\
\Sigma^{\dot{a}}(z) \Sigma^{\dot{b}}(0) \sim \frac{\epsilon^{\dot{a} \dot{b}}}{z^{1 / 2}} \\
\Sigma^{a}(z) \Sigma^{\dot{a}}(0) \sim\left(\Gamma_{M}\right)^{a \dot{a}} \psi^{M}(0) \\
\Sigma^{\dot{a}}(z) \Sigma^{a}(0) \sim\left(\Gamma_{M}\right)^{\dot{a} a} \psi^{M}(0) \\
\psi^{M}(z) \Sigma^{a}(0) \sim \Sigma^{a}(z) \psi^{M}(0) \sim \frac{1}{z^{1 / 2}}\left(\Gamma^{M}\right)^{a \dot{a}} \Sigma_{\dot{a}}(0) \\
\psi^{M}(z) \Sigma^{\dot{a}}(0) \sim \Sigma^{\dot{a}}(z) \psi^{M}(0) \sim \frac{1}{z^{1 / 2}}\left(\Gamma^{M}\right)^{\dot{a} a} \Sigma_{a}(0)
\end{gathered}
$$

The matrices $\Gamma^{M}$ have Clifford normalization. Similarly

$$
\begin{gathered}
S^{A}(z) S_{B}(0) \sim S_{A}(z) S^{B}(0) \sim \frac{\delta_{B}^{A}}{z^{3 / 4}} \\
S^{A}(z) S^{B}(0) \sim \frac{1}{z^{1 / 4}}\left(\Gamma_{\mu}\right)^{A B} \psi^{\mu}(0) \\
S_{A}(z) S_{B}(0) \sim \frac{1}{z^{1 / 4}}\left(\Gamma_{\mu}\right)_{A B} \psi^{\mu}(0) \\
\psi^{\mu}(z) S^{A}(0) \sim \frac{1}{z^{1 / 2}}\left(\Gamma^{\mu}\right)^{A B} S_{B}(0)
\end{gathered}
$$




$$
\begin{aligned}
\psi^{\mu}(z) S_{A}(0) & \sim \frac{1}{z^{1 / 2}}\left(\Gamma^{\mu}\right)_{A B} S^{B}(0) \\
S^{A}(z) \psi^{\mu}(0) & \sim \frac{1}{z^{1 / 2}} S_{B}(0)\left(\Gamma^{\mu}\right)^{B A} \\
S_{A}(z) \psi^{\mu}(0) & \sim \frac{1}{z^{1 / 2}} S^{B}(0)\left(\Gamma^{\mu}\right)_{B A}
\end{aligned}
$$

where $\left(\Gamma_{\mu}\right)_{A B}=J_{A C}\left(\Gamma_{\mu}\right)^{C D} J_{D B}$, with $J$ the antisymmetric $S p(2)$ invariant.

\section{References}

[1] L. Dixon, J. A. Harvey, C. Vafa, and E. Witten, Strings on Orbifolds, Nucl. Phys. B261 (1985) 678-686.

[2] L. Dixon, J. A. Harvey, C. Vafa, and E. Witten, Strings on Orbifolds. 2, Nucl. Phys. B274 (1986) 285-314.

[3] C. Vafa, Modular Invariance And Discrete Torsion On Orbifolds, Nucl. Phys. B273 (1986) 592.

[4] C. Vafa and E. Witten, On Orbifolds With Discrete Torsion, J. Geom. Phys. 15 (1995) 189, [hep-th/9409188].

[5] P. S. Aspinwall, D. R. Morrison, and M. Gross, Stable Singularities In String Theory, Commun. Math. Phys. 178 (1996) 115-134, [hep-th/9503208].

[6] E. R. Sharpe, Discrete Torsion And Gerbes. I, hep-th/9909108.

[7] M. R. Douglas, D-branes and Discrete Torsion, hep-th/9807235.

[8] M. R. Douglas and B. Fiol, D-branes and Discrete Torsion. Ii, hep-th/9903031.

[9] P.-M. Ho and Y.-S. Wu, Noncommutative Gauge Theories In Matrix Theory, Phys. Rev. D58 (1998) 066003, [hep-th/9801147].

[10] M. R. Douglas and G. Moore, D-branes, Quivers, and ALE Instantons, hep-th/9603167.

[11] D.-E. Diaconescu and J. Gomis, Fractional Branes and Boundary States in Orbifold Theories, hep-th/9906242.

[12] J. Maldacena, The Large-N Limit Of Superconformal Field Theories And Supergravity, Adv. Theor. Math. Phys. 2 (1998) 231-252, [hep-th/9711200].

[13] S. Kachru and E. Silverstein, 4d Conformal Theories And Strings On Orbifolds, Phys. Rev. Lett. 80 (1998) 4855, [hep-th/9802183]. 
[14] R. G. Leigh and M. J. Strassler, Exactly Marginal Operators And Duality In Four-Dimensional N=1 Supersymmetric Gauge Theory, Nucl. Phys. B447 (1995) 95-136, [hep-th/9503121].

[15] D.-E. Diaconescu, M. R. Douglas, and J. Gomis, Fractional Branes and Wrapped Branes, JHEP 02 (1998) 013, [hep-th/9712230].

[16] D. Berenstein, R. Corrado, and J. Distler, Aspects of ALE Matrix Models and Twisted Matrix Strings, Phys. Rev. D58 (1998) 026005, [hep-th/9712049].

[17] A. Hanany and A. Zaffaroni, On The Realization Of Chiral Four-Dimensional Gauge Theories Using Branes, JHEP 05 (1998) 001, [hep-th/9801134].

[18] A. Hanany and A. M. Uranga, Brane Boxes And Branes On Singularities, JHEP 05 (1998) 013, [hep-th/9805139].

[19] T. Banks, W. Fischler, S. H. Shenker, and L. Susskind, M theory as a Matrix Model: A Conjecture, Phys. Rev. D55 (1997) 5112-5128, [hep-th/9610043].

[20] R. G. Leigh and M. Rozali, Brane Boxes, Anomalies, Bending And Tadpoles, Phys. Rev. D59 (1999) 026004, [hep-th/9807082].

[21] E. Witten, Anti de Sitter Space And Holography, Adv. Theor. Math. Phys. 2 (1998) 253-291, [hep-th/9802150].

[22] S. S. Gubser, I. R. Klebanov, and A. M. Polyakov, Gauge Theory Correlators From Non-Critical String Theory, Phys. Lett. B428 (1998) 105, [hep-th/9802109].

[23] E. Witten, New 'Gauge' Theories In Six Dimensions, JHEP 01 (1998) 001, [hep-th/9710065].

[24] M. Reid, Young Person'S Guide To Canonical Singularities, Algebraic Geometry, S.J. Bloch, ed., Proc. Symp. Pure Math. 46 (1985).

[25] M. B. Green, J. A. Harvey, and G. Moore, I-brane Inflow And Anomalous Couplings On D-Branes, Class. Quant. Grav. 14 (1997) 47-52, [hep-th/9605033].

[26] L. Girardello, M. Petrini, M. Porrati, and A. Zaffaroni, Novel Local CFT And Exact Results On Perturbations Of N=4 Super Yang-Mills From Ads Dynamics, JHEP 12 (1998) 022, [hep-th/9810126].

[27] A. Connes, M. R. Douglas, and A. Schwarz, Noncommutative Geometry And Matrix Theory: Compactification On Tori, JHEP 02 (1998) 003, [hep-th/9711162].

[28] R. G. Leigh and M. Rozali, A Note On Six-Dimensional Gauge Theories, Phys. Lett. B433 (1998) 43-48, [hep-th/9712168].

[29] S. Lee, S. Minwalla, M. Rangamani, and N. Seiberg, Three-Point Functions Of Chiral Operators In D=4, N=4 Sym At Large N, Adv. Theor. Math. Phys. 2 (1998) 697-718, [hep-th/9806074]. 
[30] C. Beasley, B. R. Greene, C. I. Lazaroiu, and M. R. Plesser, D3-Branes On Partial Resolutions Of Abelian Quotient Singularities Of Calabi-Yau Threefolds, hep-th/9907186.

[31] I. R. Klebanov and E. Witten, Superconformal Field Theory On Threebranes At A Calabi-Yau Singularity, Nucl. Phys. B536 (1998) 199, [hep-th/9807080].

[32] D. R. Morrison and M. R. Plesser, Non-spherical Horizons. I, Adv. Theor. Math. Phys. 3 (1999) 1, [hep-th/9810201].

[33] A. M. Uranga, Brane Configurations For Branes At Conifolds, JHEP 01 (1999) 022, [hep-th/9811004].

[34] D. Berenstein and R. G. Leigh, work in progress.

[35] D. Berenstein and R. G. Leigh, Superstring Perturbation Theory And Ramond-Ramond Backgrounds, Phys. Rev. D60 (1999) 106002, [hep-th/9904104].

[36] D. Berenstein and R. G. Leigh, Quantization Of Superstrings In Ramond-Ramond Backgrounds, hep-th/9910145.

[37] S. Gukov, Comments On N=2 AdS Orbifolds, Phys. Lett. B439 (1998) 23, [hep-th/9806180]. 